\title{
Ensuring Efficient IT Service Management to Increase Information Systems Availability
}

\author{
Gilmar Barreto ${ }^{1}$, Paulo D. Battaglin ${ }^{1}$, Sergio Varga ${ }^{1 *}$ \\ ${ }^{1}$ Electrical Engineering and Computing Department, University of Campinas (UNICAMP), BRAZIL
}

*Corresponding Author: svarga@dsif.fee.unicamp.br

Citation: Barreto, G., Battaglin, P. D. and Varga, S. (2019). Ensuring Efficient IT Service Management to Increase Information Systems Availability. Journal of Information Systems Engineering \& Management, 4(4), em0108. https://doi.org/10.29333/jisem/6352

Published: December 9, 2019

\begin{abstract}
In today's information age the increase importance of Information Systems (IS) which are highly dependent of Information Technology (IT) forces organizations to deploy IT Service Management (ITSM) frameworks. The increase complexity of new IT technologies, products and deployment models makes the management of an IS a big challenge to manage and to ensure its availability. This complexity drives IT organizations to manage the environment by silos or technologies and inhibits a holistic view of the target environment leaving space for failures. This paper intends to analyze and identify these issues and proposes an IT management framework that will help IT organizations to provide a better management of IS.
\end{abstract}

Keywords: information systems, information technology, IT management, ITIL, ITSM

\section{INTRODUCTION}

We live in a society highly interconnected, instrumented, and dependent on computer systems. Since introduction of IT we switched from an industrial era to an information age where the ability to maintain and manage knowledge, and information, became the most important activity in organizations (Drucker, 1995). Due to the great importance of information, IS must be deployed in high-performance, high-available and high-security systems along with management strategies and models to enable an organization to be able to compete in today's world (Lastres and Albagi, 1999). The complexity of IT brings the need to have specific departments within an IT organization that will be responsible to support the operation of IS and its related IT environments.

An IS can be defined as the set of processes, users and technologies employed (hardware, software, database, networks, storage) encompassing the management of the whole organization (Turban et al., 2008). The IS and IT are directly related. The IS determine the IT needs for its operation and IT influences a change in the IS when a new technology appears (Audy et al., 2005) therefore it is imperative to implement an efficient IT management so that IS are delivered without disruptions.

The implementation of IT management is focused on the services provided and the establishment of an IT governance. This governance, aligned with the organization's business objectives provides guidelines, objectives, and metrics for implementing ITSM. There are several models or frameworks for ITSM implementation, such as ISO / IEC 20000, ISO / IEC 38500, ISO 9001, SEI CMMI, COBIT, ISO / IEC 27001 / AS7799 and Information Technology Infrastructure Library (ITIL) which this last one being the most widely used (Cater-Steel et al., 2016a; Cater-Steel et al., 2016b; Marrone and Kolbe, 2011a, 2011b).

ITIL is the most used framework for ITSM that proposes a set of best practices to help IT organizations to deploy and use IT services aligned with their business. It is structured around the life cycle of an IT service within an organization and it is structured around five services (Cartlidge et al., 2007): Service Strategy, Service Design, 
Service Transition, Service Operation and Continuous Service Improvement. Mostly of ITSM adopted implementations by large companies are based on the ITIL model in its basic concept, with some particularities. IBM implements its architecture through a process reference model (PRM-IT) and integrated by a layer that supports the lifecycle of the IS being based on four basic services: development services, business services, service management and infrastructure services (Ganek and Kloeckner, 2007). HP has its ITSM reference model structured in five areas: IT and Business Alignment, Service Design and Management, Service Delivery Certification, Service Development and Delivery and Service Operation (HP, 2003). MICROSOFT has its ITSM model, Microsoft Operations Framework (MOF), where defines a list of the processes and services needed to implement all stages of IT service management (MICROSOFT, 2008).

A study of all topics and theories for ITSM discovered that most of them are related to concepts, lacking more researches on implementations (Proehl et al., 2013). In another research the ITIL framework is verified that is proved to be able to integrate, manage and coordinate ITSM in a holistic way. The implementation of ITSM with a holistic governance and management is identified in this study (Herreza, 2017). Several other case studies (Alves and Ribeiro, 2012; Meziani and Saleh, 2010; Yao and Wang, 2010); Lucio-Nieto et al., 2012; Almeida et al., 2018; Orta and Ruiz, 2019) have done assessments in ITIL and ITSM implementations and have identified lessons learned that could improve the implementation of the service management.

In addition to these frameworks there are several other studies in this area related to deployment of ITSM frameworks or new tools. Castillo (2016) describes the aspects to perform IT operation management and project management and provide guidance to practitioners. Vorisek et al. (2015) presented a service-based IT management model that proposes a lightweight model for an effective business IT alignment as an alternative for ITIL or COBIT.

All those frameworks, architectures and studies are focus in providing guidelines to implement a solution for managing IT services. None of them are focused on how these frameworks and architectures will be deployed and managed within the IT organization through the usage of tools and people aligning with the scope of service contracted to manage IS.

This work aims to analyze and identify issues that those current frameworks and models do not cover and proposes a holistic IT management framework that will help IT organizations to provide an efficient management of IS.

\section{PROBLEM STATEMENT}

This scenario can be defined in layers as shown in Figure 1. The IS layer comprises the layer of business applications where applications are deployed to support the company's business processes. The IT layer comprises the various IT infrastructure components deployed to support those applications and it can contain a variety of products and technologies such as middleware, database, servers, storage and network. The ITSM layer comprises the IT service management disciplines deployed to support the layers above to ensure IT and IS are properly managed. Each service discipline is deployed with one or more related tool.

According to the ITSM frameworks identified above each IT service has its own controls and relationships with other services. They do not describe which tool to use or how to implement within the IT organization. In most cases they are deployed independently or in silos inhibiting the IT organization to have a holistic view of the environment to be managed.

There are many ways to increase the availability of IS such as clusters, high-availability and disaster recovery solutions. The usage of systems management tools is deployed to ensure the IT infrastructure and solutions are working properly. Also, those tools must be properly deployed and aligned with the defined scope of services (or IS) to be managed. Many ITSM disciplines must be deployed. To keep track of all managed components, from the network to the application of IS, an inventory tool is needed; to manage all components belonging to a particular client or Information System a configuration tool is needed, to make sure that all IS components are always available and that they are not impacting the business a monitoring tool is needed; to ensure that security policies are implemented and no security issues are occurring with IS, a security tool is needed; and to make sure that in the event of a disaster or need for recovery the IS can be recovery a backup tool is needed.

To support ITSM discipline and their tools IT organizations have a hierarchically structured team with technicians (support analyst, system management analyst), supervisors, managers, and executives (directors). Depending on the size of the organization this team can have professionals that perform various functions, specific or dedicated functions. This team can also be geographically distributed and work in different time zones and timetables.

As described, there are many challenges for IT organizations to provide an effective management of this complex environment. The existing ITSM models and frameworks do not focus in the tool's implementation and integration with the organization and with the scope of the IS to be managed. It is difficult to ensure all these layers 


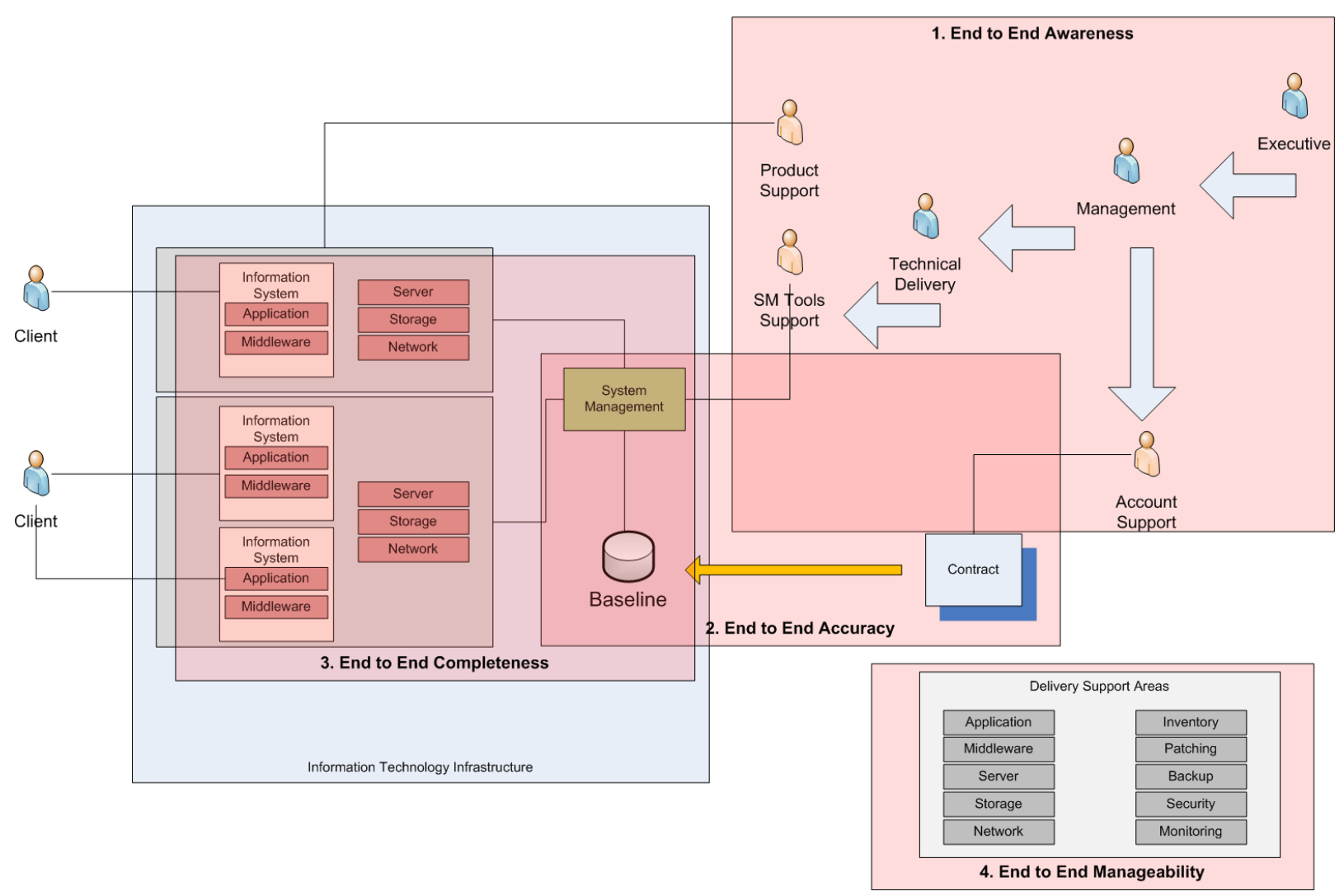

Figure 1. Information System management scenario

are integrated and managed in a holistic way. The ITSM tools and services are deployed to ensure there is no issue with the IT infrastructure that supports an IS. In an occurrence where ITSM tools are not properly working and configured can cause a failure in detecting issues in the IT infrastructure and this failure can propagate to the IS and cause an unavailability.

\section{PROBLEM IDENTIFICATION}

To increase IS availability it is important to have a holistic view englobing the IS, IT, ITSM, supporting areas and management tools as shown in Figure 2. To enable it there are four factors that are important to have it deployed with and end to end vision: awareness, accuracy, completeness and manageability (Varga et al., 2019a; Varga et al., 2019b).

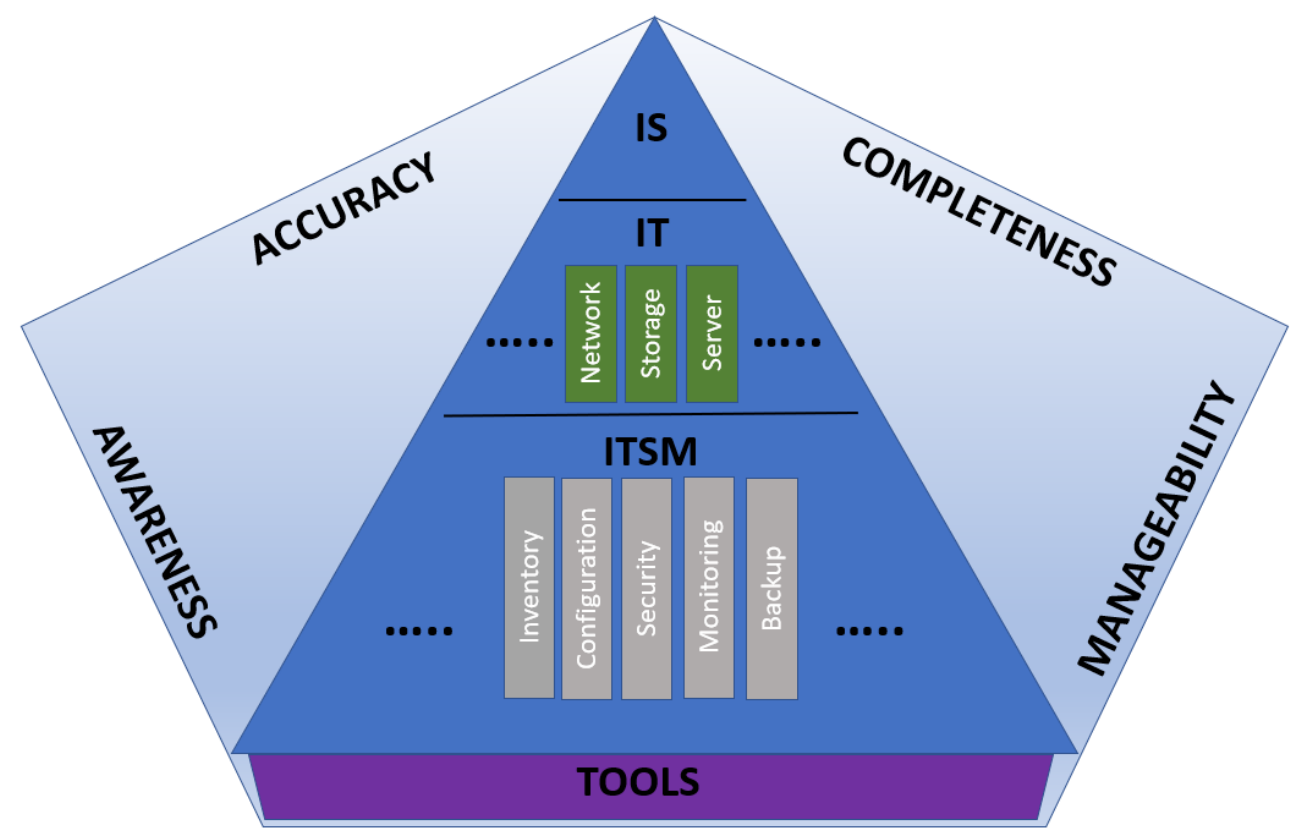

Figure 2. ITSM holistic view

(C) 2019 by Author/s 
Awareness of the environment to be managed by the entire IT organization team, from the low-level systems IT analysts up to the executive level encompassing the various IT support areas, departments and management layers. An effective understanding of the environment across the entire IT organization will enable it to deliver an effective management of the IS and its IT infrastructure ensuring a best service through the synergy between the internal areas of the IT organization. The lack of a good understanding of the managed IS by the IT organization will limit and make the delivery of the service prone to errors, as it can lead to failures in communication between the different teams that manage the environment. It also may inhibit the professional to be proactive and agile, either not alerting or not identifying some impact from another area in the overall health of the environment.

Accuracy of the IS (or scope) to be managed and its IT components. It describes the IS, its components (servers and applications), the levels of service and availability to be delivered, and which contracted IT services was selected. All these definitions must be accurately understood by the IT organization and ITSM and tools must be deployed. The lack of proper management of an IS component, for example monitoring of a critical server, can cause unavailability of the IS and consequently a financial problem or loss to the organization. It is important to ensure all IS and IT components are identified and the ITSM and tools are deployed for the entire environment.

Completeness related to the systems management tools implemented, making sure that they are properly managing all the identified components related to the IS. These management tools are those defined by the IT organization and include tools for managing all IT components, server capacity and performance, monitoring and alerting problems, backup and recovery, security, configuration and inventory, management of problems, incidents, changes and availability. All these tools and services will ensure the IS availability. Failing to ensure all systems management tools are properly implemented in all components may cause the failure in capturing issues in IS components that can impact its availability.

Manageability of various systems management services and tools (problems, incidents, changes, availability, backup, capacity, performance, security, and inventory) with the IT supporting areas, ensuring that all ITSM tools are working and reporting properly. Failure to report issues with the tools can affect availability of IS when, for instance, a database monitoring agent is not working and do not identify that the database is stopped impacting the IS. The tools deployed to support ITSM must always be working properly accordingly to the IS defined.

It is necessary to apply a holistic view when deploying ITSM and its tools aligning the management of IS with the IT organization, so that everyone responsible for this administration will have the same level of knowledge about what is happening with the IS and its IT environment. By leveraging this holistic view all stakeholders within the IT organization can proactively act in an event of failure and minimize future problems in the IS increasing its availability.

\section{DESIGNED SOLUTION}

This research will develop a framework which will addresses the issues identified related to these four factors to provide a holistic view and be able to increase IS availability. The framework will be implemented in a three-tier architecture solution composed by a collection layer, logic layer and presentation layer as described in Figure 3.

The collection layer will collect the status of all ITSM services managed and report the integrity of the tools deployed by the organization. The logic layer will verify the contracted ITSM services and IS's client infrastructure, validate and build a scope of services per client and perform the holistic validation with the deployed status of ITSM tools. The presentation layer will allow the stakeholders to have a holistic view about all the ITSM service status health and their clients.

The main feature for solving the awareness problem is centered on the presentation layer. It will allow any employee (from all levels of organization) to verify any problem in the IT infrastructure regardless of allocation to the given service. The awareness problem should be solved by the framework by making available the status of all ITSM services provided to the entire IT organization responsible for the IS and IT infrastructure. With this, any employee will be aware, at any time, of problems in the environment or failure of service provided, regardless of acting in any area of support, and be able to promptly work in the resolution.

The problem of accuracy is handled by the logic layer. The resolution of this problem starts with the identification of all available services, the correct identification of what IT services were contracted, and the verification of was contracted versus the services available. The result of this verification should reflect the correct configuration of the scope to be administered by the IT organization. This scope is the basis for implementing the contracted IT services and configuring the deployed ITSM tools. Failures identified in this verification will be reported for further correction by the IT organization.

The problem of completeness is related to the fact that not all systems management tools are fully implemented for all contracted services, and it is therefore important that each area responsible for these tools uses the scope file generated in the scope validation process. The completeness problem should be solved by the framework making sure that the systems management tools are implemented in all IT infrastructures, that supports the IS. 


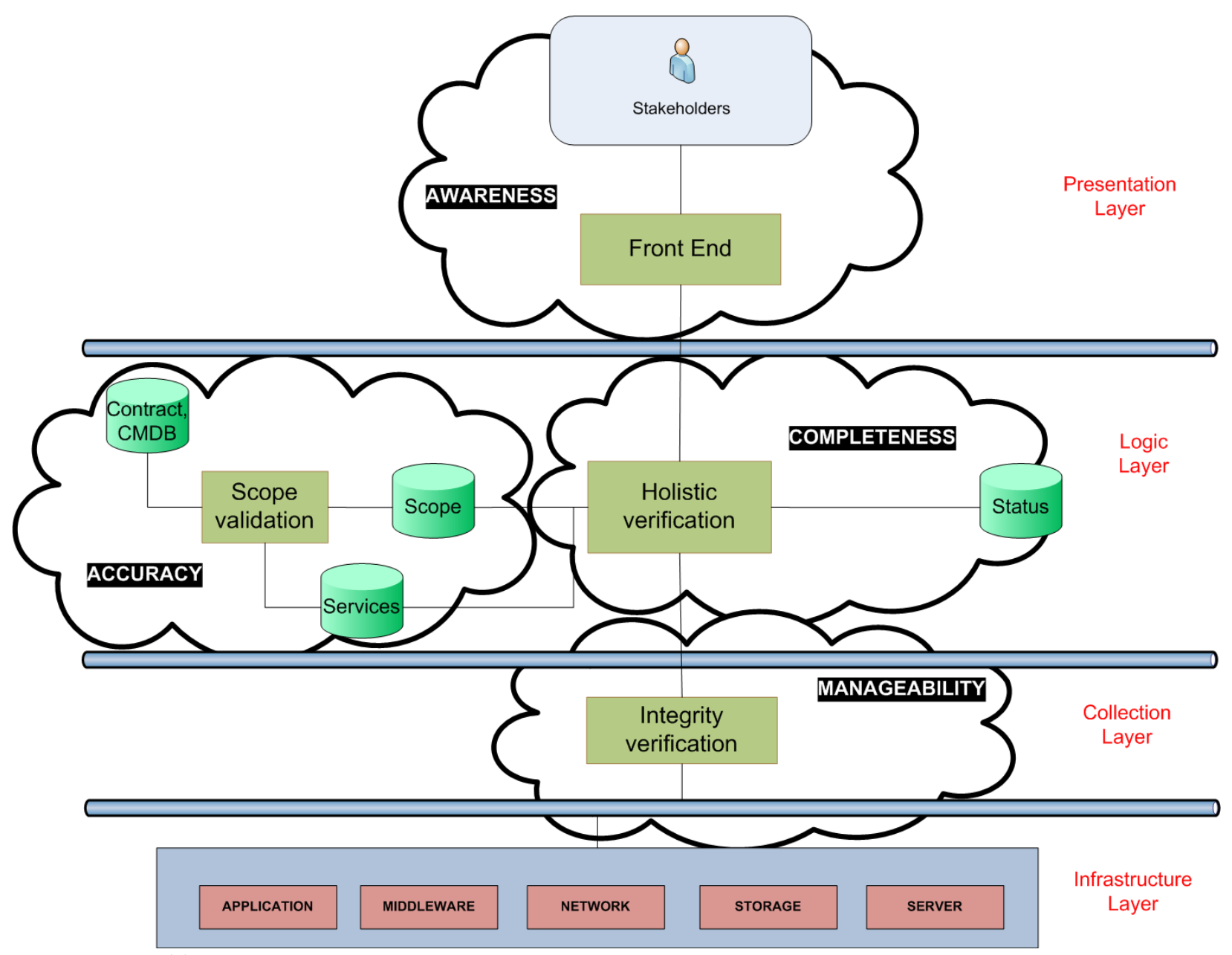

Figure 3. Architecture

The problem of manageability is to ensure that the implemented ITSM tools are working without failures and that are identified and reported according to the current state of the managed environment. This problem is solved thought the collection layer by doing an integrity check of the current state of the implemented ITSM tools, ensuring that these tools are implemented without failures and reporting the errors for the supporting organization to fix it.

\section{CONCLUSION AND FUTURE WORK}

As described in this paper there are a couple of IT infrastructure solutions and components available to enable or increase IS availability. In addition to it there are ITSM frameworks and architectures available to ensure the IS and IT infrastructure will be always available. Tools must be deployed for each ITSM discipline defined to support the IS and IT and these set of tools are managed by the IT organization and must be deployed according to the managed IS.

After extensive research it was identified that besides all existing ITSM frameworks and architectures there is no solution that focus to ensure a holistic view that handles IS, IT, ITSM, supporting areas and management tools. It is important to have an end to end vision of this environment to be managed and align with the IT organization. To enable it is important to ensure four factors: awareness, accuracy, completeness and manageability.

The major contribution of this research is to propose a holistic IT management framework that will handle the problems identified in this research ensuring those four factors are covered. We expect this can contribute with the IS studies and help increase IS availability through an efficient implementation and management of ITSM.

The next steps of this research are to develop a prototype that can show the framework presented in this study will solve the problems identified and addresses the four issues. Then apply this prototype in a production IT environment to validate the proposed research brings the expected benefits by solving the four identified issues that inhibits the increase of IS availability. 


\section{REFERENCES}

Almeida, T., De Vasconcelos, J. B. and Pestana, G. (2018). A knowledge management architecture for information technology services delivery. Proceedings of 13th Iberian Conference on Information Systems and Technologies (CISTI), 1316 June, Caceres, Spain, 390-393, IEEE. https://doi.org/10.23919/CISTI.2018.8399202

Alves, V. and Ribeiro, P. C. J. (2012). Information Technology governance - A case study of the applicability of ITIL and COBIT in a Portuguese Private School. Proceedings of 7 th Iberian Conference on Information Systems and Technologies (CISTI), 20-23 June, Madrid, Spain, 382-387, IEEE.

Audy, J. L. N., Andrade, G. K. and Cifral, A. (2005). Fundamentos de Sistemas de Informação. Porto Alegre: Bookman.

Cartlidge, A., Hanna, A., Rudd, C., Macfarlane, I., Windebank, J. and Rance, S. (2007). An Introductory Overview of ITIL V3, itSMF UK.

Castillo, F. (2016). Managing Information Technology. Springer International Publishing. Available at: https://link.springer.com/book/10.1007/978-3-319-38891-5

Cater-Steel, A., Toleman, M. and Wui-Gee, T. (2016a). Summary Report of ITSM Standards and Frameworks Survey, Technical Report, University of Southern Queensland, Toowoomba, Australia. Available at: http://eprints.usq.edu.au/7875/1/Cater-Steel_Tan_Toleman_Report_2009_AV.pdf (Accessed 20 March 2016).

Cater-Steel, A., Toleman, M., Valverde, R. and Shrestha, A. (2016b). Decision support systems for IT service management. International Journal of Information and Decision Sciences, 8(3), 284-304. https://doi.org/10.1504/IJIDS.2016.078588

Drucker, P. F. (1995). Managing in a time of great change. New York: Truman Talley Books/Dutton.

Ganek, A. and Kloeckner, K. (2007). An Overview of IBM Service Management. IBM Systems Journal, 46(3), 375385. https://doi.org/10.1147/sj.463.0375

Herreza, P. C. (2017). Innovation in Information Technology Services: Framework to Improve the Effectiveness and Efficiency of Information Technology Service Management Processes, Projects and Decision Support Management, World Academy of Science, Engineering and Technology. International Journal of Social, Behavioral, Educational, Economic, Business and Industrial Engineering, 11(8), 2037-2044. https:// doi.org/10.5281/zenodo.1131679

HP (2003). The HP IT Service Management (ITSM) Reference Model. Available at: ftp://ftp.hp.com/pub/services/itsm/info/itsm_rmwp.pdf (Accessed 10 October 2017).

Lastres, H. M. M. and Albagi, S. (1999). Informação e Globalização na Era do Conhecimento. Rio de Janeiro: Campus.

Lucio-Nieto, T., Colomo-Palacios, R., Soto-Acosta, P., Popa, S. and Amescua-Seco, A. (2012). Implementing an IT service information management framework: The case of COTEMAR. International Journal of Information Management, 32(6), 589-594. https://doi.org/10.1016/j.ijinfomgt.2012.08.004

Marrone, M. and Kolbe, L.M. (2011a). Uncovering ITIL claims: IT executive's perception on benefits and Business - IT alignment. International Systems and E-business Management, 9(3), 363-380. https://doi.org/10.1007/s10257010-0131-7

Marrone, M. and Kolbe, L.M. (2011b). Impact of IT Service Management Frameworks on the IT Organization. Business \& Information Systems Engineering, 3(1), 5-18. https://doi.org/10.1007/s12599-010-0141-5

Meziani, R. and Saleh, I. (2010). e-government: ITIL-based service management case study. Proceedings of the 12th International Conference on Information Integration and Web-based Applications \& Services, 08-10 November, Paris, France, 509-516. https://doi.org/10.1145/1967486.1967565

MICROSOFT (2008). Microsoft Operations Framework Overview 4.0. Available at: http://download.microsoft.com/ download/6/5/8/658bc1e9-e262-45ca-bb6e-e87c058bbd37/microsoft\%20operations\%20framework\%204 .zip (Accessed 10 July 2017).

Orta, E. and Ruiz, M. (2019). Met4ITIL: A process management and simulation-based method for implementing ITIL. Computer Standards \& Interfaces, 61, 1-19. https:// doi.org/10.1016/j.csi.2018.01.006

Proehl, T., Erek, K., Limbach, F. and Zarnekow, R. (2013). Topics and applied theories in it service management. Proceedings of 46th Hawaii International Conference on System Sciences, 7-10 January, Hawaii, USA, 1367-1375. https:// doi.org/10.1109/HICSS.2013.555

Turban, E., Leidner, D., McLean, E. and Wetherbe, J. (2008). Information Technology for Management: Transforming Organizations in the Digital Economy". New Jersey: Wiley.

Varga, S., Barreto, G. and Battaglin, P. D. (2019a). A Holistic IT infrastructure system management framework. International Journal of Computational Science and Engineering, in press. https://doi.org/10.1504/IJCSE.2019.10018360

Varga, S., Barreto, G. and Battaglin, P. D. (2019b). Increasing Information Systems Availability through Accuracy, Awareness, Completeness and Manageability of ITSM. Proceedings of 14th Iberian Conference on Information Systems and Technologies (CISTI), 19-22 June, Coimbra, Portugal, IEEE. https://doi.org/10.23919/CISTI.2019.8760686 
Vorisek, J., Pour, J. and Buchalcevova, A. (2015). Management of business informatics model: principles and practices. E\&M Ekonomie a Management, 3, 160-173. http://dx.doi.org/10.15240/tul/001/2015-3-014

Yao, Z. and Wang, X. (2010). An ITIL based ITSM practice: A case study of steel manufacturing enterprise. Proceedings of 2010 7th International Conference on Service Systems and Service Management, 28-30 June, Tokyo, 1-5, IEEE. https://doi.org/10.1109/ICSSSM.2010.5530204 